\title{
Hyaluronan negatively regulates vascular calcification involving BMP2 signaling
}

\author{
Yonglun Kong ${ }^{1,2,3} \cdot$ Qingchun Liang ${ }^{4} \cdot$ Yanting Chen ${ }^{1,2,3} \cdot$ Pingzhen Yang ${ }^{1,2,3} \cdot$ Xiaoyu Liu ${ }^{1,2,3}$. \\ Yining $\mathrm{Li}^{1,2,3} \cdot$ Siyuan Feng ${ }^{1,2,3} \cdot \mathrm{Ji} \mathrm{Wu}^{1,2,3} \cdot$ Wantao $\mathrm{Liu}^{1,2,3} \cdot$ Jingyi Tang ${ }^{1,2,3} \cdot$ Huimin $\mathrm{Yu}^{5}$.

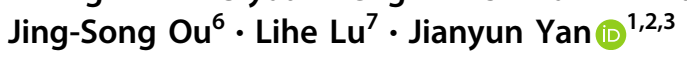

Received: 31 January 2018 / Revised: 21 March 2018 / Accepted: 16 April 2018 / Published online: 21 May 2018

(c) United States \& Canadian Academy of Pathology 2018

\begin{abstract}
Vascular calcification is a highly regulated biological process similar to bone formation involving osteogenic differentiation of vascular smooth muscle cells (VSMCs). Hyaluronan (HA), a major structural component of the extracellular matrix in cartilage, has been shown to inhibit osteoblast differentiation. However, whether HA affects osteogenic differentiation and calcification of VSMCs remains unclear. In the present study, we used in vitro and ex vivo models of vascular calcification to investigate the role of HA in vascular calcification. Both high and low molecular weight HA treatment significantly reduced calcification of rat VSMCs in a dose-dependent manner, as detected by alizarin red staining and calcium content assay. Ex vivo study further confirmed the inhibitory effect of HA on vascular calcification. Similarly, HA treatment decreased ALP activity and expression of bone-related molecules including Runx2, BMP2 and Msx2. By contrast, inhibition of HA synthesis by 4-methylumbelliferone (4MU) promoted calcification of rat VSMCs. In addition, adenovirus-mediated overexpression of HA synthase 2 (HAS2), a major HA synthase in VSMCs, also inhibited calcification of VSMCs, whereas CRISPR/Cas9-mediated HAS2 knockout promoted calcification of rat A10 cells. Furthermore, we found that BMP2 signaling was inhibited in VSMCs after HA treatment. Recombinant BMP2 enhanced high calcium and phosphate-induced VSMC calcification, which can be blocked by HA treatment. Taken together, these findings suggest that HA inhibits vascular calcification involving BMP2 signaling.
\end{abstract}

These authors contributed equally: Yonglun Kong, Qingchun Liang, Yanting Chen.

Electronic supplementary material The online version of this article (https://doi.org/10.1038/s41374-018-0076-x) contains supplementary material, which is available to authorized users.

\footnotetext{
Lihe Lu

lulihe@mail.sysu.edu.cn

$\triangle$ Jianyun Yan

yanjy790@163.com

1 Department of Cardiology, Laboratory of Heart Center, Heart Center, Zhujiang Hospital, Southern Medical University, Guangzhou, China

2 Guangdong Provincial Biomedical Engineering Technology Research Center for Cardiovascular Disease, Guangzhou, China

3 Sino-Japanese Cooperation Platform for Translational Research in
}

\section{Introduction}

Vascular calcification is a very common pathologic condition in chronic kidney diseases [1,2]. Vascular calcification contributes to hemodynamic imbalance and decrease of vessel wall elasticity, thus increasing cardiovascular morbidity and mortality among patients with chronic kidney diseases. In the past, vascular calcification

\section{Heart Failure, Guangzhou, China}

Department of Anesthesiology, The Third Affiliated Hospital, Southern Medical University, Guangzhou, China

5 Department of Cardiology, Guangdong General Hospital, Guangdong Academy of Medical Sciences and Guangdong Cardiovascular Institute, Guangzhou, China

6 Division of Cardiac Surgery, The First Affiliated Hospital, Sun Yat-Sen University, Guangzhou, China

7 Department of Pathophysiolgy, Zhongshan Medical School, Sun Yat-Sen University, Guangzhou, China 
was regarded as a passive and degenerative process with precipitation of calcium and phosphate in arteries. Accumulating studies have shown that vascular calcification is a highly regulated biological process involving osteogenic differentiation of vascular smooth muscle cells (VSMCs) similar to bone formation, characterized by the upregulation of bone-related proteins including Runx2 and BMP2, and downregulation of contractile proteins including SMA and SM22 $\alpha[3,4]$. Elevated calcium and phosphate levels are important factors promoting vascular calcification in chronic kidney disease [5-7]. However, the precise molecular mechanisms underlying vascular calcification are not fully understood.

Extracellular matrix (ECM) plays important roles in a variety of cellular physiological process including cell differentiation, survival and proliferation. A number of studies have demonstrated that ECM molecules including matrix Gla protein, osteoprotegerin and decorin are important regulators of vascular calcification [8-11]. Hyaluronan (HA), a liner polysaccharide consisting of repeated disaccharide units of D-glucuronate and $\mathrm{N}$-acetyl-D-gluco-samine, is a main structural component of the ECM. HA synthase 2 (HAS2) is the major HA synthase responsible for HA synthesis in cultured VSMCs $[12,13]$. Conditional knockout (KO) of HAS2 study reveals that HAS2-mediated production of HA is required for bone formation and chondrocyte maturation [14]. HA is known to participate in the regulation of many cellular activities including cell proliferation, cell migration and osteoclastogenesis [15-17]. Recent studies have demonstrated that HA promotes the embryonic stem cell differentiation toward a smooth muscle cell lineage, and inhibits osteoblast differentiation [18, 19], but another study suggests that HA prmotes osteoblast differentiation [20]. However, it is unclear whether HA regulates osteogenic differentiation of VSMCs and vascular calcification.

Bone morphogenetic protein-2 (BMP2), a member of the transforming growth factor beta (TGF- $\beta$ ) superfamily, has been identified as one of the most important molecules to regulate bone formation and osteoblast differentiation [21]. It has been reported that BMP2 expression is increased in VSMCs during vascular calcification [22]. Both in vitro and in vivo studies have demonstrated that BMP2 promotes vascular calcification [23, 24]. However, the link between HA and BMP2 during vascular calcification remains elusive. In this study, we used in vitro and ex vivo models of vascular calcification to investigate the role of $\mathrm{HA}$ in vascular calcification and the link between HA and BMP2 signaling during vascular calcification.

\section{Materials and methods}

\section{Cell culture}

All animal experiments were performed in accordance with the US National Institutes of Health guidelines and were approved by the Institutional Animal Care and Use Committee at Southern Medical University. VSMCs were isolated from aortic arteries of Sprague-Dawley (SD) rats (200-220 g) using explant method as described previously [11]. Rat VSMC line A10 was purchased from the American Type Culture Collection (Manassas, USA). All cell culture reagents were purchased from Life Technology Company, USA. VSMCs were grown in Dulbecco's modification of Eagle's medium (DMEM) supplemented with $10 \%$ fetal bovine serum (FBS), 100 units/ml of penicillin and $100 \mathrm{mg} /$ $\mathrm{ml}$ of streptomycin at $37^{\circ} \mathrm{C}$ with $5 \% \mathrm{CO}_{2}$. Cells between passages five and eight were used for all the experiments. Calcifying medium (CM; DMEM supplemented with $10 \mathrm{mM}$ beta-glycerophosphate (BGP) and $3 \mathrm{mM} \quad \mathrm{CaCl}_{2}$ ) was used to induce VSMC calcification [25]. To investigate the role of HA in vascular calcification, HA (R\&D System, USA) was used to treat cells in CM for 6 days or 10 days at different concentrations (50, 100 or $200 \mu \mathrm{g} / \mathrm{ml})$. In all, $0.25 \mu \mathrm{M}$ of 4-methylumbelliferone (4MU, Sigma), a HA synthesis inhibitor, was used to treat VSMCs in some experiments. VSMCs were treated with recombinant human BMP2 at $100 \mathrm{ng} / \mathrm{ml}$ in the presence of CM for 10 days. All in vitro experiments were performed independently three times.

\section{Aortic ring organ culture}

Thoracic aortas were isolated from SD rats and cut into segments of $0.5 \mathrm{~cm}$ in length. To induce arterial calcification, aortic segments were incubated in $\mathrm{CM}$ for 8 or 10 days at $37^{\circ} \mathrm{C}$ in a humidified $5 \% \mathrm{CO}_{2}$ incubator. $\mathrm{HA}(200 \mu \mathrm{g} / \mathrm{ml})$ was used to treat aortic segments in CM for 8 days or 10 days. The organ culture experiment was performed independently four times.

\section{Cell transfection}

Cells were transfected with adenovirus encoding HAS2 (Ad/HAS2) or control virus (Ad/GFP) as described previously $[11,26]$. Cells were seeded in $35 \mathrm{~mm}$ dishes at $4 \times 10^{5}$ cells/dish and grown in DMEM supplemented with $10 \%$ FBS. When cells reached $80 \%$ confluence, Ad/HAS2 or Ad/GFP were used to infect cells at an optimal multiplicity of infection $(\mathrm{MOI}=10)$ and the transfection efficiency reaches $70 \%$. Transfected cells were harvested at indicated time points for further analysis. 


\section{CRISPR/Cas9-mediated HAS2 KO in A10 cells}

Knockdown of HAS2 in A10 cells was performed by CRISPR/Cas9-guided genome editing. Two of 20 nucleotide sgRNA sequences: gRNA1 5'-ATGCAACTAAATACA CGGCT-3' (23263-23282) and gRNA2 5'-CCTTGATCTA TGCGTGCTAC-3' (23884-23903) were designed using sgRNA CRISPR design tool online (http://crispr.mit.edu) and cloned into the pSpCas9(BB)-2A-Puro (PX459) plasmid (Addgene, catalog no. 62988). After cloning, plasmids were purified and verified by sequencing. Rat A10 VSMCs were seeded into six-well plates at $70 \%$ confluence and transfected with PX459 plasmid encoding a target specific sgRNA using Lipofectamine 3000 (Invitrogen) according to the manufacturer's instructions. Puromycin was then added to select the transfected cells after $24 \mathrm{~h}$ transfection. KO efficiency for HAS2 was assessed by western blot.

\section{Determination of calcification}

Alizarin red staining was used to detect cell and arterial calcification at indicated time points. For cell culture, VSMCs grown in $35 \mathrm{~mm}$ dishes were fixed in $4 \%$ formaldehyde for $10 \mathrm{~min}$ and incubated with $2 \%$ alizarin red ( $\mathrm{pH} 4.2$ ) for $5 \mathrm{~min}$ at room temperature. To remove the excess dye, cells were washed with deionized water. Alizarin red dye was eluted with $10 \%$ formic acid and quantified by spectrophotometry. For aortic ring culture, aortic segments were fixed in $4 \%$ paraformaldehyde (PFA) and embedded in paraffin. Aortic samples were cut into $6 \mu \mathrm{m}$ in thickness. Sections were deparaffinized followed by staining with $2 \%$ alizarin red. Images were taken using an inverted microscope. Calcium content was measured using o-cresolphthalein complexone method as previously described [27]. VSMCs were washed with phosphate-buffered saline (PBS) and incubated with $0.6 \mathrm{~N} \mathrm{HCl}$ for $24 \mathrm{~h}$. The aortic segments were dried and weighed, followed by incubation in $0.6 \mathrm{~N} \mathrm{HCl}$ for decalcification. Protein concentration was quantified using $\mathrm{BCA}^{\mathrm{TM}}$ protein assay (Pierce, USA). Calcium content was expressed as $\mu \mathrm{g} / \mathrm{mg}$ protein or $\mu \mathrm{g} / \mathrm{mg}$ dry tissue.

\section{ALP activity assay}

Alkaline phosphatase (ALP) activity was assessed as previously described $[11,25]$. Briefly, VSMCs were harvested with $0.1 \%$ Triton X-100 in PBS at indicated time points. Protein samples were then mixed with paranitrophenylphosphate (p-NPP) $(180 \mu \mathrm{l})$ substrate and incubated for $15 \mathrm{~min}$ at $37^{\circ} \mathrm{C}$. After the reaction was stopped by $\mathrm{NaOH}$, absorbance was then measured at $405 \mathrm{~nm}$. Protein concentration was measured using $\mathrm{BCA}^{\mathrm{TM}}$ protein assay (Pierce, USA). ALP activity was expressed as $\mathrm{nmol} / \mathrm{ml} \mathrm{p}$ nitrophenol converted per microgram of protein per minute.

\section{HA ELISA}

HA levels in the media of cell culture were determined using HA Elisa kit (SBJ-R0037, Nanjing SenBeiJia Biological Technology, China) according to the manufacturer's instructions. A HA standard curve generated was linear between 22.5 and $270 \mathrm{ng} / 1$ of the HA standard supplied by the manufacturer. HA levels were normalized to control.

\section{Quantitative real-time PCR}

Total RNA was extracted from cells using TRIzol regents (Invitrogen, USA) according to the manufacturer's instructions. Reverse transcription kit (Takara Company, Japan) was used to synthesize complementary DNA. Quantitative realtime PCR (qPCR) was performed using SYBR Green mixture (Takara Company, Japan) in a StepOne Plus real-time PCR system (Applied Biosystems, USA). PCR primers were as follows: $\beta$-actin (forward): 5'-TGTCACCAACTGGGACGA TA-3', $\beta$-actin (reverse): 5'-GGGGTGTTGAAGGTCTCAA A-3'; Runx2 (forward): 5'-GCCGGGAATGATGAGAA CTA-3', Runx2 (reverse): 5'-GGACCGTCCACTGTCACT TT-3'; BMP2 (forward): 5'-GTTTGGCCTGAAGCAGAGA C-3', BMP2 (reverse): 5'-CTCGATGGCTTCTTCGT GAT-3'; Msx2 (forward): 5'-CCTCGGTCAAGTCGGA AAAT-3', Msx2 (reverse): 5'-ACTGTTTCTGGCGGAAC TTG-3'. Value was normalized to $\beta$-actin and relative gene expression was determined using comparative $\mathrm{Ct}$ method.

\section{Western blot analysis}

Total protein was extracted from cultured VSMCs and protein concentration was determined by a BCA protein assay kit. Proteins were separated by sodium dodecyl sulfate polyacrylamide gel electrophoresis and transferred to polyvinylidene fluoride membranes (Millipore, USA). The membranes were then blocked with 5\% nonfat milk for $1 \mathrm{~h}$ and incubated with primary antibodies including BMP2 (1:2000, Abcam, USA), Runx2 (1:2000, Abcam, USA), pSmad1/5/8 (1:1000, Cell Signaling Technology, USA), Smad1 (1:2000, ABclonal Biotechnology, USA), HAS2 (1:1000, Abcam, USA), Akt, p-Akt, extracellular signalregulated kinase (ERK), phosphorylated extracellular signal-regulated kinase (p-ERK) (1:1000, Cell Signaling Technology, USA), $\beta$-actin (1:3000, Cell Signaling Technology, USA) overnight at $4{ }^{\circ} \mathrm{C}$. Then, the membranes were washed and incubated with horseradish peroxidaseconjugated secondary antibodies (EarthOx Life Sciences, USA) for $1 \mathrm{~h}$. Protein signals were detected by Lumazone Chemiluminescent Substrate (Millipore, USA). Protein expression levels were quantified by densitometry and normalized to $\beta$-actin expression. 
A

$\mathrm{CM}$

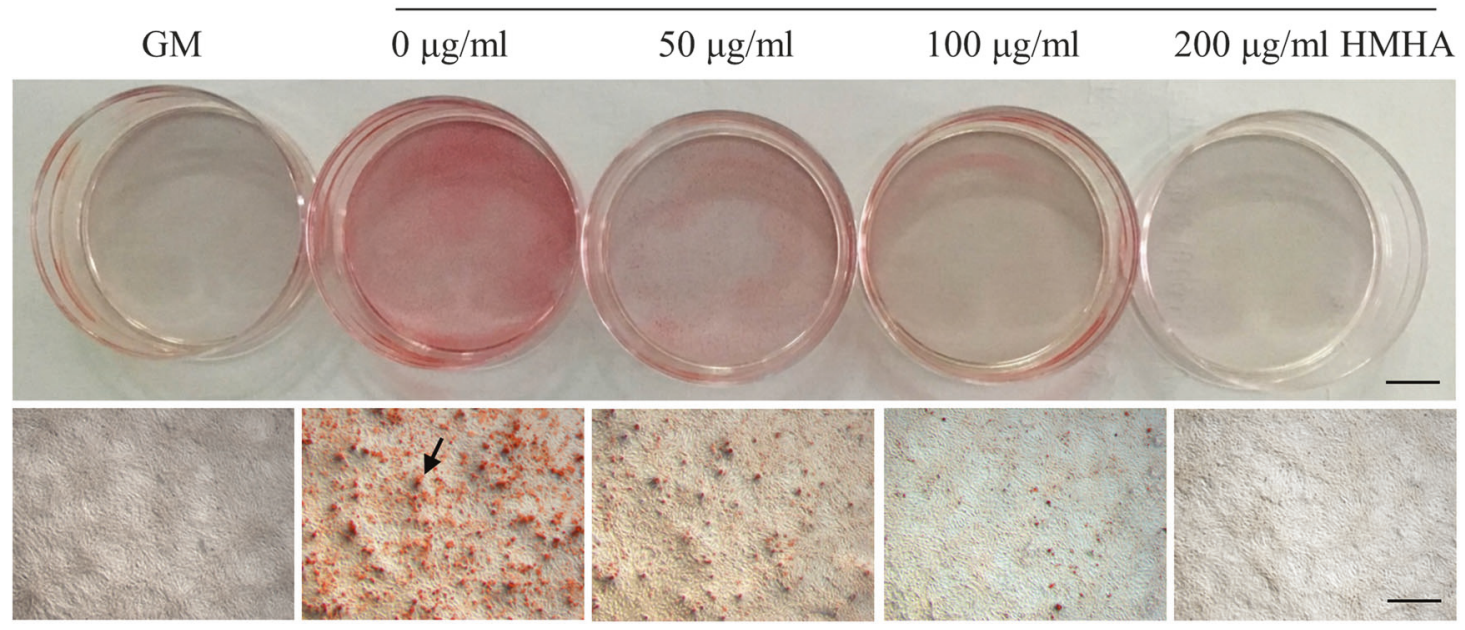

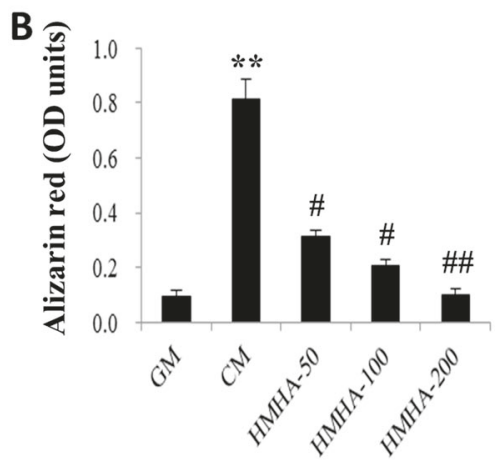

E

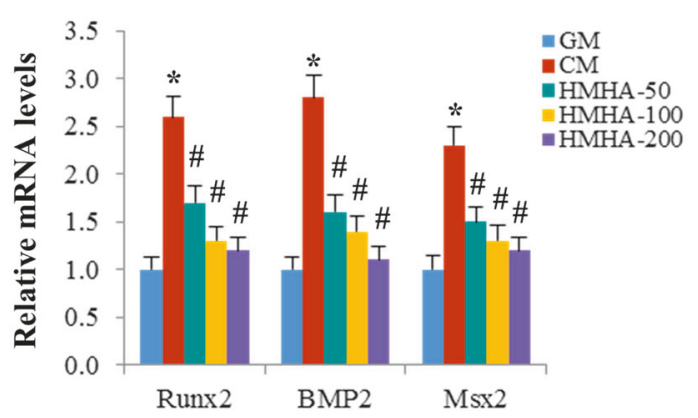

Fig. 1 Effect of High molecular weight-hyaluronan on rat VSMC calcification. Confluent rat VSMCs were cultured in growth medium $(\mathrm{GM})$, calcifying medium (CM) or $\mathrm{CM}$ supplemented with high molecular weight-hyaluronan (HMHA) for 10 days $(n=3)$. a Mineral deposition in VSMCs was detected by alizarin red staining at day 10 . Scale bar $=200 \mu \mathrm{m}$. b Alizarin red dye was eluted with $10 \%$ formic
$\mathbf{F}$

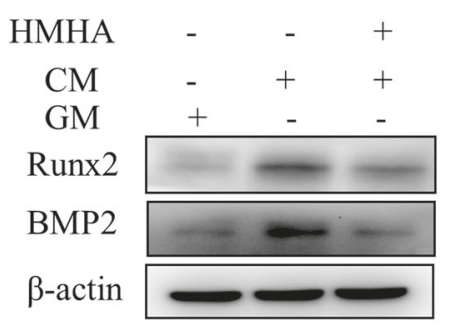

acid and quantified by a microplate reader. c Calcium content was measured at day 10 . d ALP activity was assessed by spectrophotometry at day 6. e Runx2, BMP2, and Msx2 mRNA levels were determined by qPCR at day 6 . $\mathbf{f}$ The protein expression of Runx 2 and BMP2 was analyzed by western blot at day $6 . * P<0.05$, $* * P<0.01$ vs. GM; ${ }^{\#} P<0.05,{ }^{\# \#} P<0.01$ vs. $\mathrm{CM}$

\section{Immunocytochemistry}

Rat VSMCs were cultured in chamber slides, fixed in $4 \%$ paraformaldehyde and permeabilized with $0.5 \%$ Triton X100 for $15 \mathrm{~min}$ at room temperature. After being blocked with $10 \%$ goat serum in PBS at room temperature for $1 \mathrm{~h}$, cells were incubated with antibody for p-Smad1/5/8 in 5\% bovine serum albumin solution overnight at $4{ }^{\circ} \mathrm{C}$. Cells were washed and then incubated with Alexa Fluor-594conjugated goat anti-rabbit secondary antibody for $1 \mathrm{~h}$ at room temperature. The nuclei were counterstained in blue with 4, 6-diamidino-2-phenylindole (DAPI, Molecular Probes). The images were visualized and photographed with a fluorescent microscope (LEICA, DM4000B). 
A

$\mathrm{CM}$

GM $\quad 5 \mu \mathrm{g} / \mathrm{ml} \quad 50 \mu \mathrm{g} / \mathrm{ml} \quad 100 \mu \mathrm{g} / \mathrm{ml} \quad 200 \mu \mathrm{g} / \mathrm{ml} \mathrm{LMHA}$

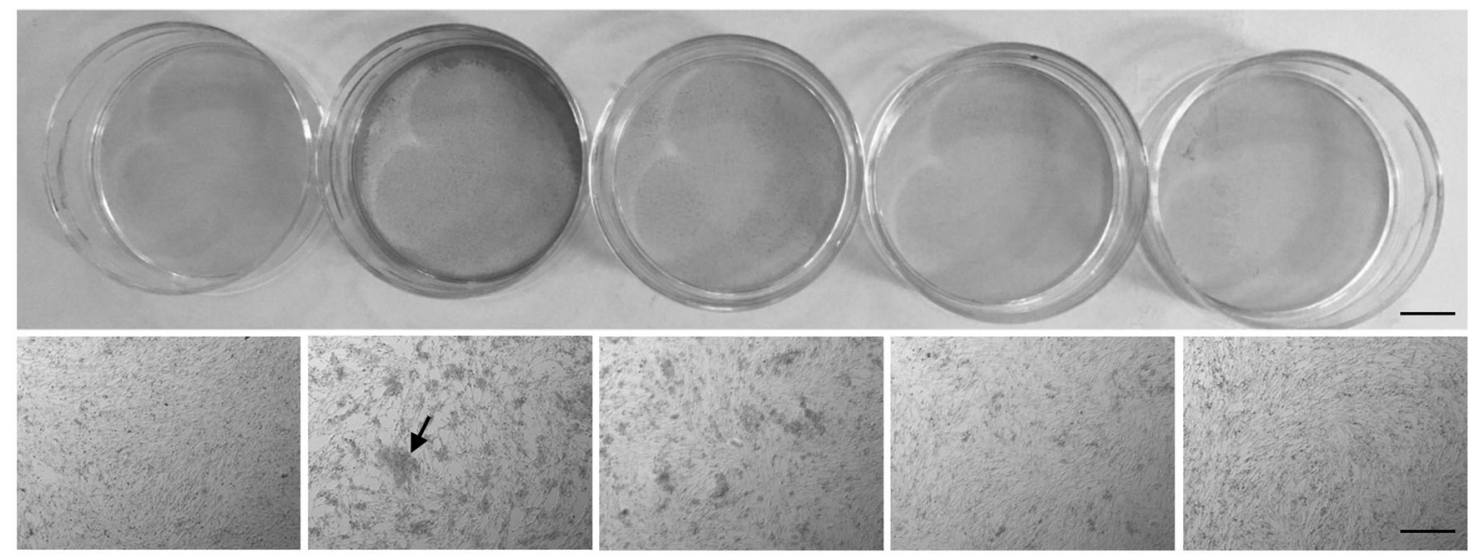

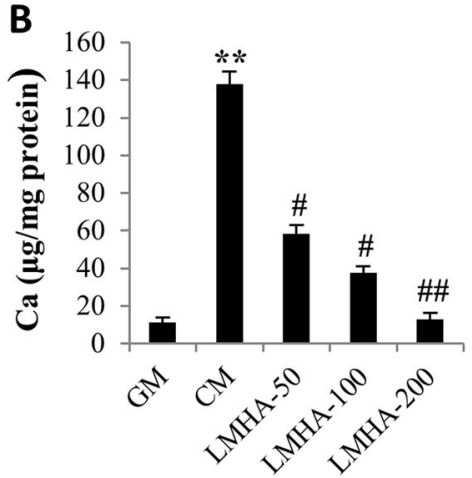

Fig. 2 Effect of low molecular weight-hyaluronan on rat VSMC calcification. Confluent rat VSMCs were cultured in growth medium (GM), calcifying medium (CM) or $\mathrm{CM}$ supplemented with low molecular weight-hyaluronan (LMHA) for 10 days $(n=3)$. a Mineral deposition in VSMCs was detected by alizarin red staining at day 10 .
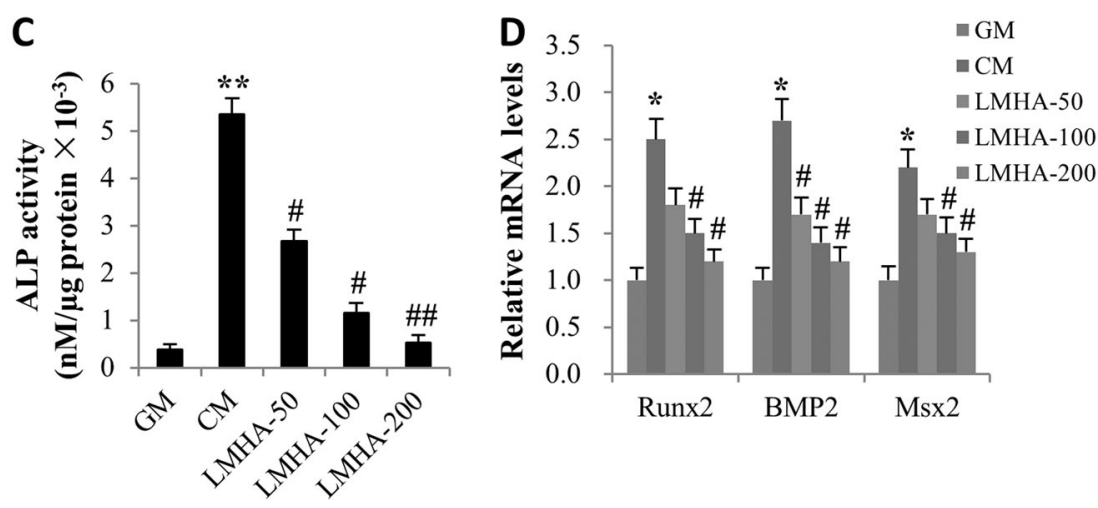

\section{Statistical analysis}

All data are expressed as mean \pm SD from three independent experiments and analyzed using the software package SPSS 17.0. Statistical differences between two groups were analyzed by Student's $t$-test and differences between more than two groups were compared by one-way analysis of variance. A value of $P<0.05$ was considered statistically significant.

\section{Results}

\section{High molecular weight HA inhibits calcification of rat VSMCs}

To investigate the effect of HA on calcification of rat VSMCs, CM was used to stimulate VSMC calcification and
Scale bar $=200 \mu \mathrm{m}$. b Calcium content was measured at day 10. c ALP activity was assessed by spectrophotometry at day 6. d Runx2, BMP2, and Msx $2 \mathrm{mRNA}$ levels were determined by qPCR at day 6 . $* P<0.05,{ }^{* *} P<0.01$ vs. GM; ${ }^{\#} P<0.05,{ }^{\# \#} P<0.01$ vs. $\mathrm{CM}$

rat VSMCs were treated with 50,100 or $200 \mu \mathrm{g} / \mathrm{ml}$ of high molecular weight HA (HMHA). Calcium deposition was observed in CM-treated rat VSMCs, but not in growth medium (GM)-treated cells, as indicated by alizarin red staining. HMHA reduced mineral deposition in a dosedependent manner in the presence of $\mathrm{CM}$ at day 10 (Figs. 1a, b). Calcium content assay further confirmed that calcium content was reduced by $66 \%(P<0.05), 83 \%(P<$ $0.05), 91 \%(P<0.01)$ in $50,100,200 \mu \mathrm{g} / \mathrm{ml}$ of HMHAtreated cells, respectively, compared with CM-treated cells (Fig. 1c). Next, we examined the effect of HMHA on osteogenic differentiation of rat VSMCs. The activity of $\mathrm{ALP}$, an early osteogenic differentiation marker, was significantly decreased in HMHA-treated cells at day 6, compared with cells treated without HMHA in CM (Fig. 1d). Additionally, qPCR analysis showed that HMHA reduced mRNA expression of bone-related molecules including Runx2, BMP2, and Msx2 in VSMCs at day 6 (Fig. 1e). 
A
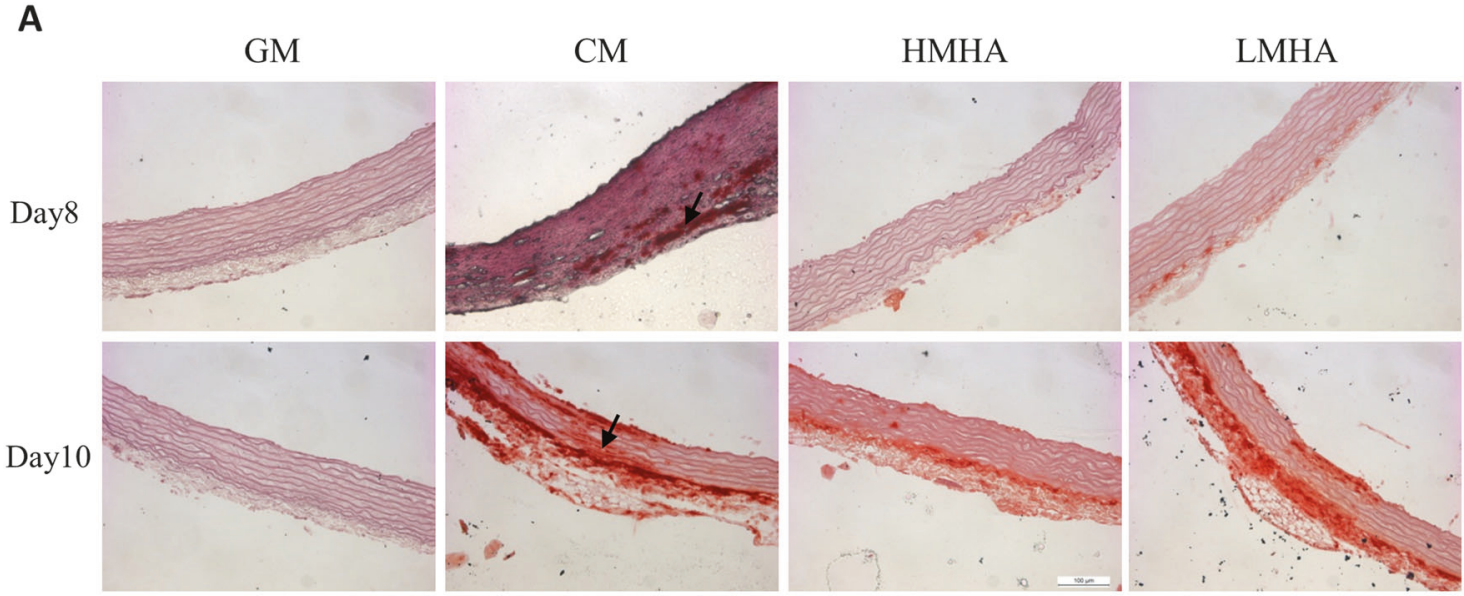

B

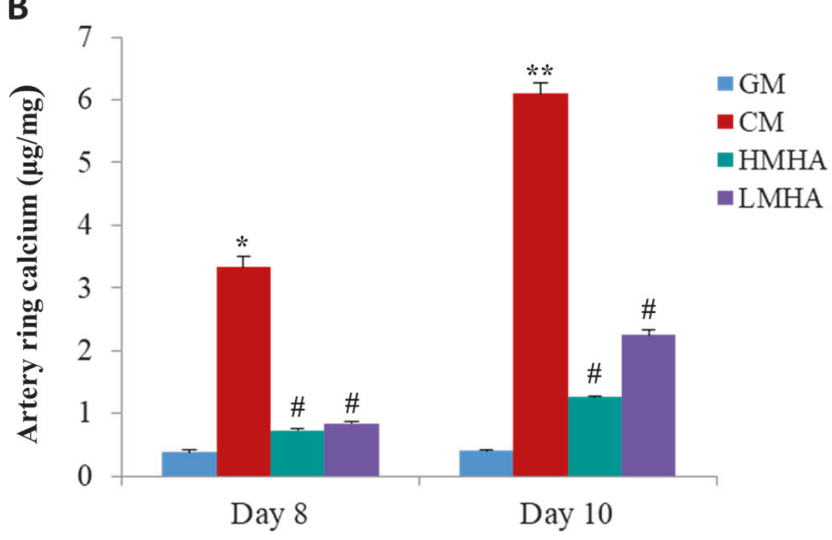

Fig. 3 Effect of hyaluronan on calcification of rat aortic ring. Rat aortic ring were cultured in growth medium (GM), calcifying medium (CM) or $\mathrm{CM}$ supplemented with high molecular weight-hyaluronan (HMHA) or low molecular weight-hyaluronan (LMHA) for 10 days. a

Mineral deposition in aortas was detected by alizarin red staining at day 8 and $10(n=4)$. Scale bar $=100 \mu \mathrm{m}$. b Calcium content was measured at day 8 and $10 .{ }^{*} P<0.05,{ }^{*} P<<0.01$ vs. GM; ${ }^{\#} P<0.05$ vs. $\mathrm{CM}$

Western blot analysis confirmed the downregulation of Runx2 and BMP2 protein expression by HMHA treatment (Fig. 1f).

\section{Low molecular weight HA inhibits calcification of rat VSMCs}

To further verify the effect of HA on VSMC calcification, we also used low molecular weight HA (LMHA) to treat rat VSMCs. Alizarin red staining showed that LMHA reduced calcium deposition in a dose-dependent manner in the presence of $\mathrm{CM}$ at day 10 (Fig. 2a). LMHA-treated cells showed less levels of calcium content than CM-treated cells (Fig. 2b). In addition, we found that LMHA inhibited osteogenic differentiation of rat VSMCs, as indicated by the reduction of ALP activity and downregulation of Runx2, BMP2 and Msx2 mRNA expression in VSMCs at day 6 (Figs. 2c, d).

\section{HA attenuates calcification of rat aortic ring}

To determine the effect of HA on vascular calcification ex vivo, we used HMHA and LMHA to treat rat aortic ring. Alizarin red staining revealed that both HMHA and LMHA reduced CM-induced calcium deposition in aortic arteries at day 8 and day 10 (Fig. 3a). Quantification of calcium content analysis further confirmed that HMHA reduced calcium content by $78 \%$ and $80 \%(P<0.05)$ in aortic arteries at day 8 and day 10, respectively (Fig. 3b). Similarly, LMHA reduced calcium content by $75 \%$ and $63 \%(P$ $<0.05)$ in aortic arteries at day 8 and day 10 , respectively (Fig. 3b).

\section{Inhibition of HA synthesis promotes calcification of rat VSMCs}

Next, we examined the effect of $4 \mathrm{MU}$, a competitive inhibitor of HA biosynthesis, on VSMC calcification. Alizarin 
A

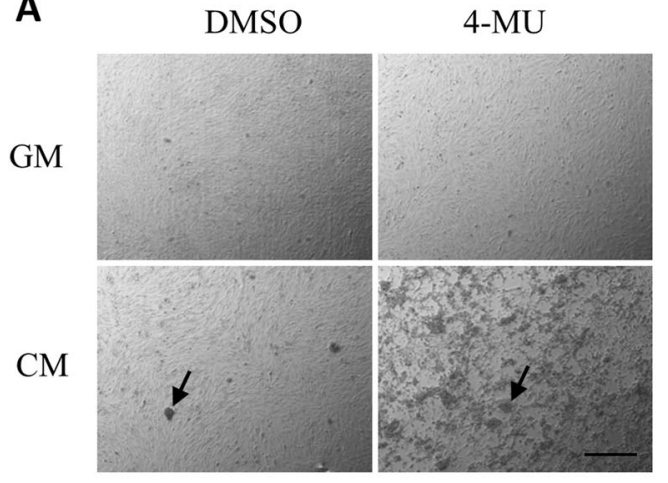

C

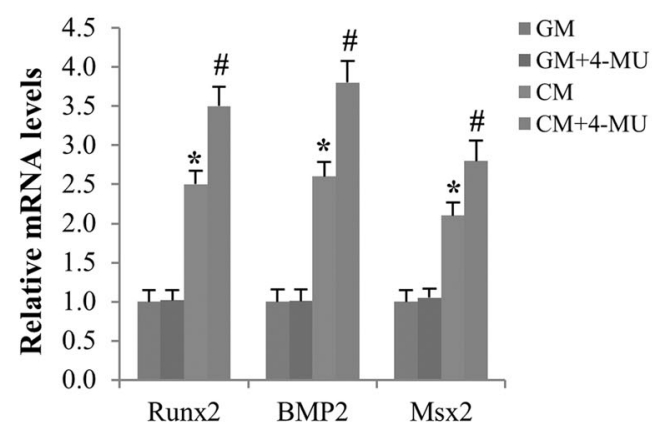

B


$=200 \mu \mathrm{m}$. b Calcium content was measured. c Runx2, BMP2, and Msx 2 mRNA levels were determined by qPCR. d ALP activity was assessed by spectrophotometry. ${ }^{*} P<0.05$ vs. GM; ${ }^{\#} P<0.05$, ${ }^{\# \#} P<0.01$ vs. $\mathrm{CM}$

controls (Fig. 5a). Mineral deposition was reduced by $89 \%$ $(P<0.05)$ in Ad/HAS2-infected cells compared with controls, which was confirmed by calcium content assay (Figs. 5b, c). Moreover, HAS2 overexpression significantly reduced ALP activity by $90 \%(P<0.05)$ in rat VSMCs (Fig. 5d). These data suggest a key role of HAS2 in regulating osteogenic differentiation and calcification of VSMCs.

\section{KO of HAS2 accelerates calcification of rat VSMCs}

To further validate the role of HAS2 in VSMC calcification, we performed KO of HAS2 in rat VSMC line A10 using CRISPR/Cas9-guided genome editing. Western blot analysis confirmed decreased levels of HAS2 protein in HAS2 KO cells compared with control cells (Fig. 6a). KO of HAS2 accelerated mineral deposition in VSMCs (Fig. 6b) and obviously increased the levels of calcium deposition by 3.9-fold $(P<0.05$, Fig. 6c). Similarly, ALP activity was also increased by 2.9 -fold $(P<0.05)$ in HAS2 KO cells compared with controls (Fig. 6d). 
A
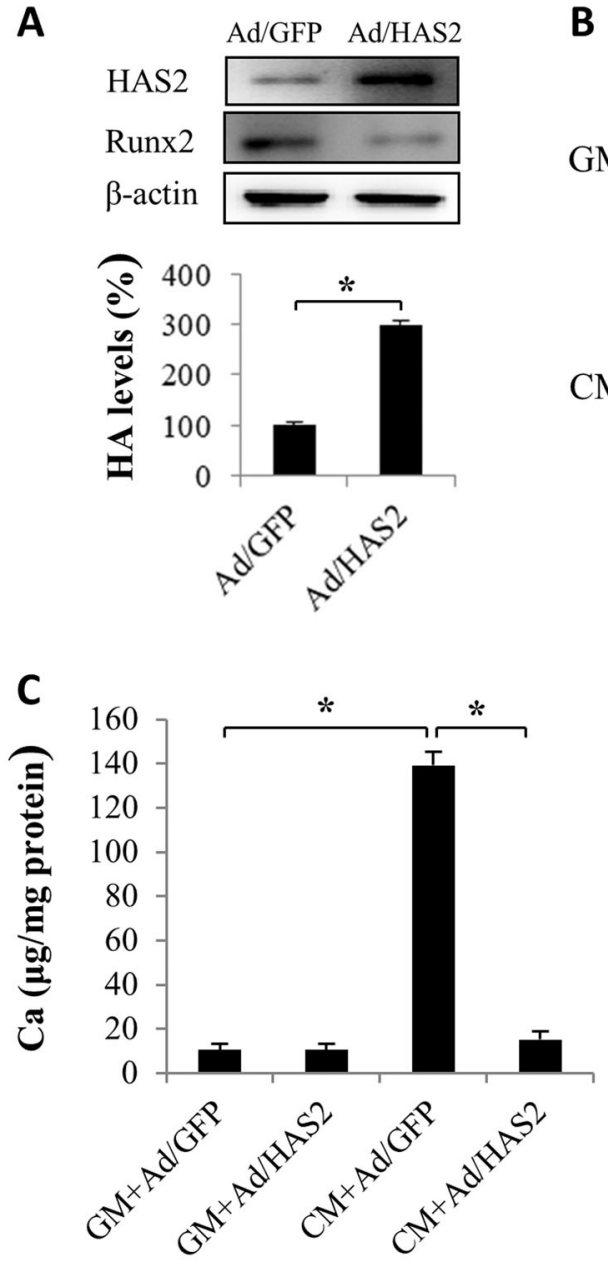

Fig. 5 Effect of HAS2 overexpression on rat VSMC calcification. Rat VSMCs were transfected with Ad/GFP or Ad/HAS2 $(n=3)$. a The protein expression of HAS2 was analyzed by western blot and the levels of HA were assessed by Elisa at day 3. b Mineral deposition in

\section{HA regulates BMP2 signaling in rat VSMCs}

Previous studies have demonstrated that BMP2 is an important positive regulator of vascular calcification [24, 28]. Therefore, we then investigated the effect of HA on BMP2 signaling in VSMCs. Western blot analysis revealed that BMP2 and downstream signaling molecules p-Smad1/ $5 / 8$ were highly expressed in CM-treated cells compared with controls, and both HMHA and LMHA treatment can downregulate protein expression of BMP2 and inhibit phosphorylation of Smad1/5/8 (Fig. 7a). In contrast, 4MU treatment upregulated BMP2 protein expression and potentiated phosphorylation of Smad1/5/8 (Fig. 7b). Immunocytochemistry analysis confirmed that $\mathrm{CM}$ treatment stimulated phosphorylation of Smad1/5/8, and $4 \mathrm{MU}$ treatment potentiated phosphorylation of Smad1/5/8, but this effect was inhibited by HMHA treatment (Fig. 7c). In addition, we examined the effect of HA on the activation of
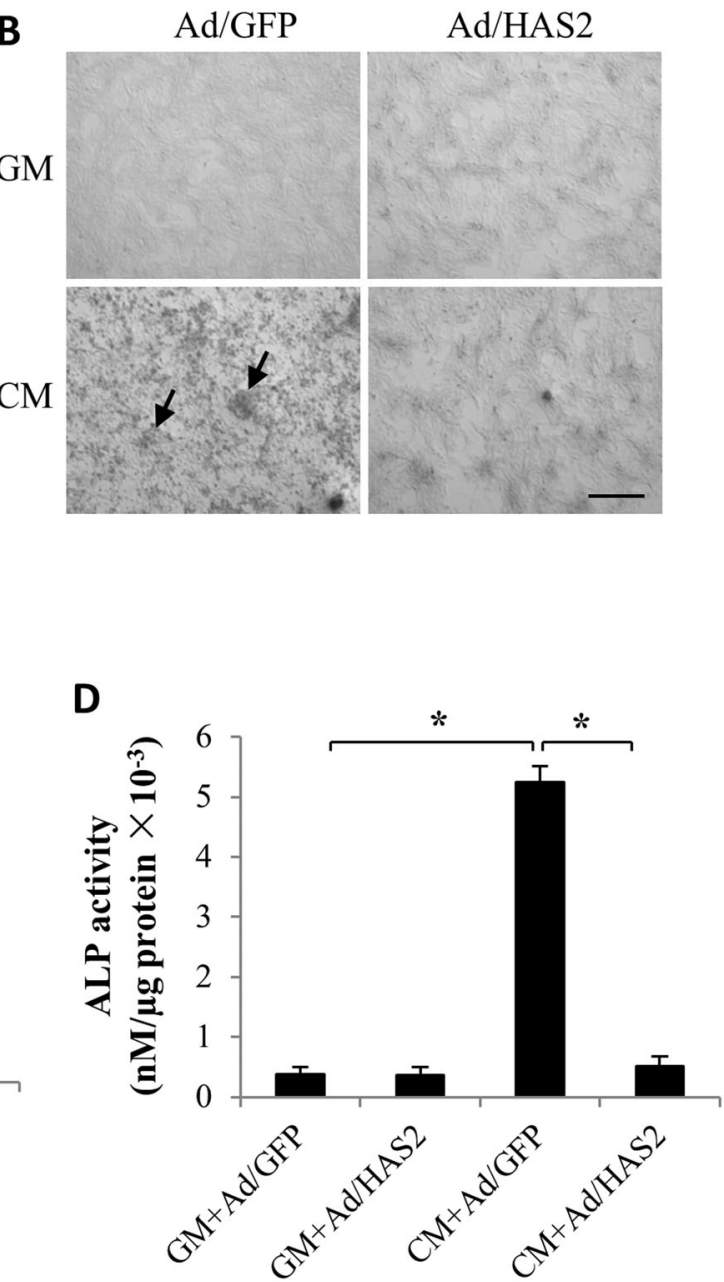

VSMCs was detected by alizarin red staining at day 10 . Scale bar = $200 \mu \mathrm{m}$. c Calcium content was measured at day 10. d ALP activity was assessed by spectrophotometry at day $6 .{ }^{*} P<0.01$

p-Smad1/5/8 in VSMCs induced by recombinant BMP2. Immunocytochemistry analysis showed that HA treatment partially reduced the phosphorylation of Smad1/5/8 induced by recombinant BMP2 (Supplementary Fig. 1). Taken together, these results suggest a potential role of BMP2 signaling in regulation of VSMC calcification inhibition by HA.

\section{BMP2 signaling is required for VSMC calcification inhibition by $\mathrm{HA}$}

To further determine whether BMP2 signaling is required for VSMC calcification inhibition by HA treatment, recombinant BMP2 protein was used to treat cells in CM for 10 days. We found that recombinant BMP2 protein treatment enhanced CM-induced mineral deposition in VSMCs, which can be blocked by both HMHA and LMHA treatment. More mineral deposition was detected in BMP2 
A
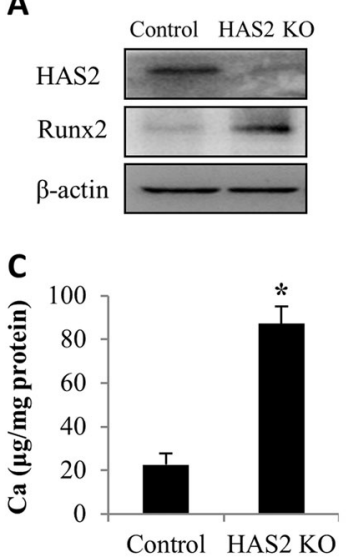

B

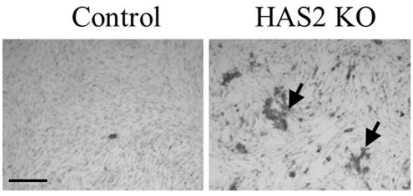

D

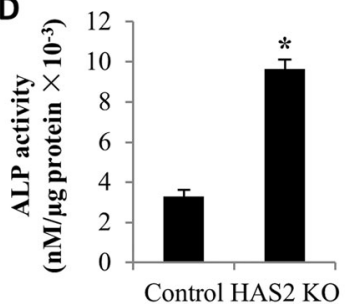

Fig. 6 Effect of HAS2 knockout on VSMC calcification. Confluent rat A10 VSMCs were transfected with PX459 plasmid encoding a target specific sgRNA using Lipofectamine $3000(n=3)$. a The protein expression of HAS2 was analyzed by western blot. b Mineral deposition in VSMCs was detected by alizarin red staining at day 10 . Scale bar $=200 \mu \mathrm{m}$. c Calcium content was measured at day 10 . d ALP activity was assessed by spectrophotometry at day $6 .{ }^{*} P<0.05$ vs. control

together with HMHA-treated cells than HMHA-treated cells in the presence of CM, suggesting inhibitory effect of HA on VSMC calcification can be reversed by BMP2 treatment (Fig. 8a). Similarly, BMP2 treatment significantly increased the levels of calcium content, and this effect was prevented by HA treatment. The levels of calcium content were increased in BMP2 together with HMHA-treated cells compared with HMHA-treated cells in the presence of CM (Fig. 8b). Additionally, ALP activity in rat VSMCs was enhanced by BMP2 treatment in the presence of CM, which can be blocked by HA treatment (Fig. 8c). These results suggest that BMP2 signaling plays an important role in regulation of VSMC calcification by HA. Given that Akt and ERK signaling pathways play important roles in vascular calcification [29, 30], we examined the effect of HA on Akt and ERK signaling pathways using Ad/HAS2 and 4MU. Western blot analysis showed both HAS2 overexpression and $4 \mathrm{MU}$ have no significant effect on the phosphorylated levels of Akt and ERK (Supplementary Fig. 2), suggesting that HA does not affect on Akt and ERK signaling pathways.

\section{Discussion}

Vascular calcification is now considered as an active generegulated process resembling osteogenesis [31, 32]. Therefore, it is very important to decipher the molecular mechanisms underlying vascular calcification, so that therapeutic strategies can be designed to prevent and treat vascular calcification. In this study, we demonstrate that both HMHA and LMHA treatment reduces calcification of

rat VSMCs and arterial calcification. Similarly, HA treatment prevents osteogenic differentiation of VSMCs. By contrast, inhibition of HA synthesis by 4MU promotes calcification of rat VSMCs. In addition, HAS2 overexpression inhibits calcification of VSMCs, whereas HAS2 KO promotes calcification of rat A10 cells. Furthermore, BMP2 signaling is required for the inhibitory effect of HA on VSMC calcification. To our knowledge, this is the first report to show that HA inhibits vascular calcification involving BMP2 signaling.

A growing evidence has demonstrated the importance of glycosaminoglycan chains for the regulation of mineralization [33, 34]. Previous studies have shown that oversulfated chondroitin sulfate promotes osteoblast differentiation and mineralization [35] and decorin glycosaminoglycan chain regulates human VSMC calcification [11]. HA is a glycosaminoglycan polymer component of the ECM. An animal study has revealed that inhibition of HA synthesis by $4 \mathrm{MU}$ accelerates atherosclerosis in $\mathrm{ApoE}^{-1-}$ mice [36]. HA has been shown to inhibit osteoblast differentiation [18] and the calcification-mitigating role of the HA grafts on the biomaterial has been demonstrated [34]. In this study, we find that HMHA inhibits osteogenic differentiation and calcification of rat VSMCs and aterial calcification. The molecular mass of HA ranges from $10^{5}$ to $10^{7}$ $\mathrm{Da}$ and HA function depends on the length of the polysaccharide chains. Different forms of HA have distinct effects on cellular function. For instance, HMHA inhibits VSMC cycle progression, whereas LMHA stimulates cell cycle progression [15]. Therefore, we also investigated the effect of LMHA on vascular calcification in this study. Interestingly, LMHA also reduces calcification of rat VSMCs and aterial calcification, suggesting that the molecular mass of HA is not the major determinant affecting vascular calcification under the conditions of high calcium and phosphate. These findings further confirmed the inhibitory effect of HA on vascular calcification.

HAS2 is the major enzyme responsible for HA synthesis in VSMCs [12] and has been shown to regulate atherosclerosis [37]. In this study, we show that overexpression of HAS2 inhibits calcification of rat VSMCs, whereas $\mathrm{KO}$ of HAS2 accelerates calcification of rat VSMCs, suggesting that HAS2 plays important role in vascular calcification. Previous studies have demonstrated that BMP2 regulates osteoblast differentiation through osteogenic transcription factors including Runx2 and Msx2 [38, 39]. Several studies have revealed that BMP2 positively regulates vascular calcification [23, 24]. Inhibition of BMP signaling reduces vascular calcification [40] and HA has been shown to inhibit BMP2-induced osteoblast differentiation [18]. Therefore, we investigated whether HA negatively regulates vascular calcification involving BMP2 signaling. We find that HA treatment downregulates protein expression of 
A

A

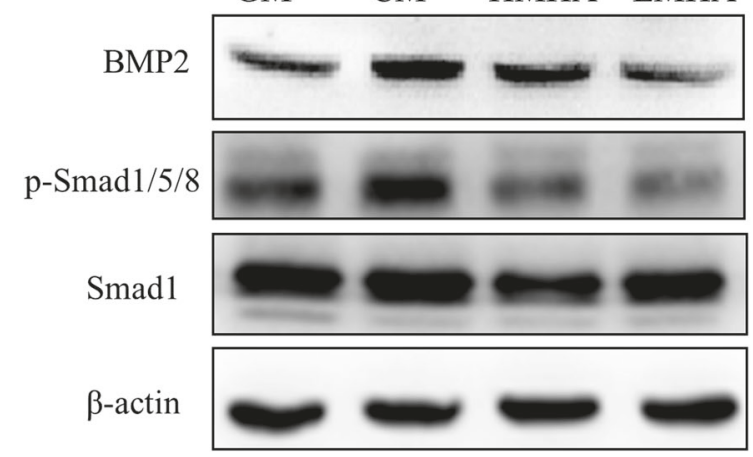

B

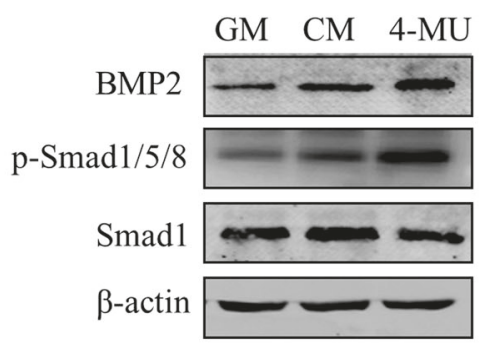

C
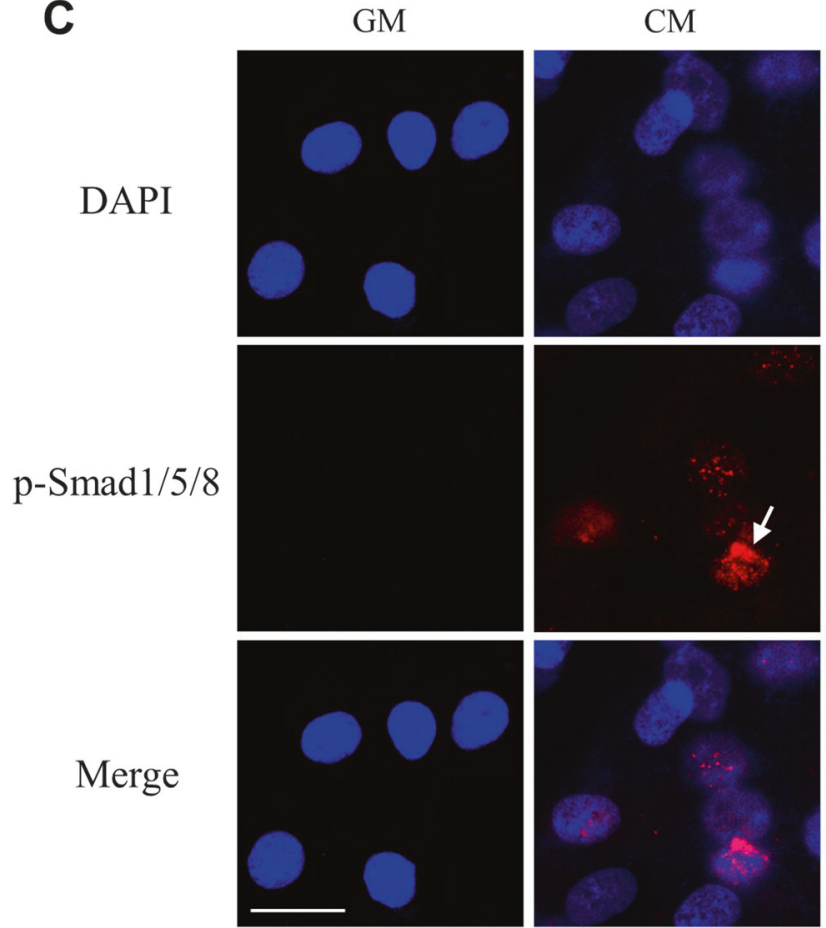
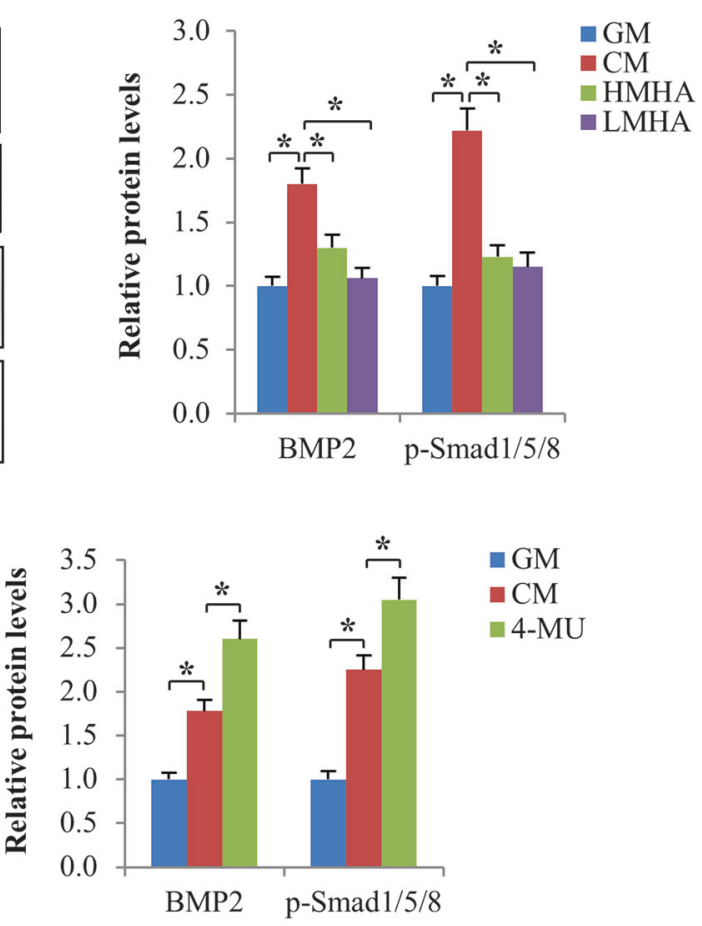

BMP2 p-Smad1/5/8


Fig. 7 Effect of HA on BMP2 signaling in rat VSMCs. Confluent rat VSMCs were cultured in growth medium (GM), calcifying medium (CM) or CM supplemented with HMHA, LMHA or 4MU for 6 days

BMP2 and inhibits phosphorylation of Smad1/5/8. Conversely, inhibition of HA synthesis by $4 \mathrm{MU}$ upregulates BMP2 protein expression and potentiates phosphorylation of Smad1/5/8. Importantly, inhibitory effect of HA on $(n=3)$. a, b The protein expression of BMP2, Smad1 and p-Smad1/5/ 8 was analyzed by western blot. c The levels of p-Smad1/5/8 were analyzed by immunostaining. $* P<0.05$

VSMC calcification can be reversed by BMP2 treatment and BMP2-induced VSMC calcification was inhibited by HA treatment, suggesting the key role of BMP2 in HAregulated vascular calcification. 
Fig. 8 Effect of BMP2 on rat VSMC calcification. Confluent rat VSMCs were cultured in growth medium (GM), calcifying medium (CM) or $\mathrm{CM}$ supplemented with BMP2, HMHA, BMP2 with HMHA, or BMP2 with LMHA for 10 days $(n=3)$. a Mineral deposition in VSMCs was detected by alizarin red staining at day 10 . Scale bar $=200 \mu \mathrm{m}$. b Calcium content was measured at day 10. c ALP activity was assessed by spectrophotometry at day $6 . * P$ $<0.05, * * P<0.01$
A
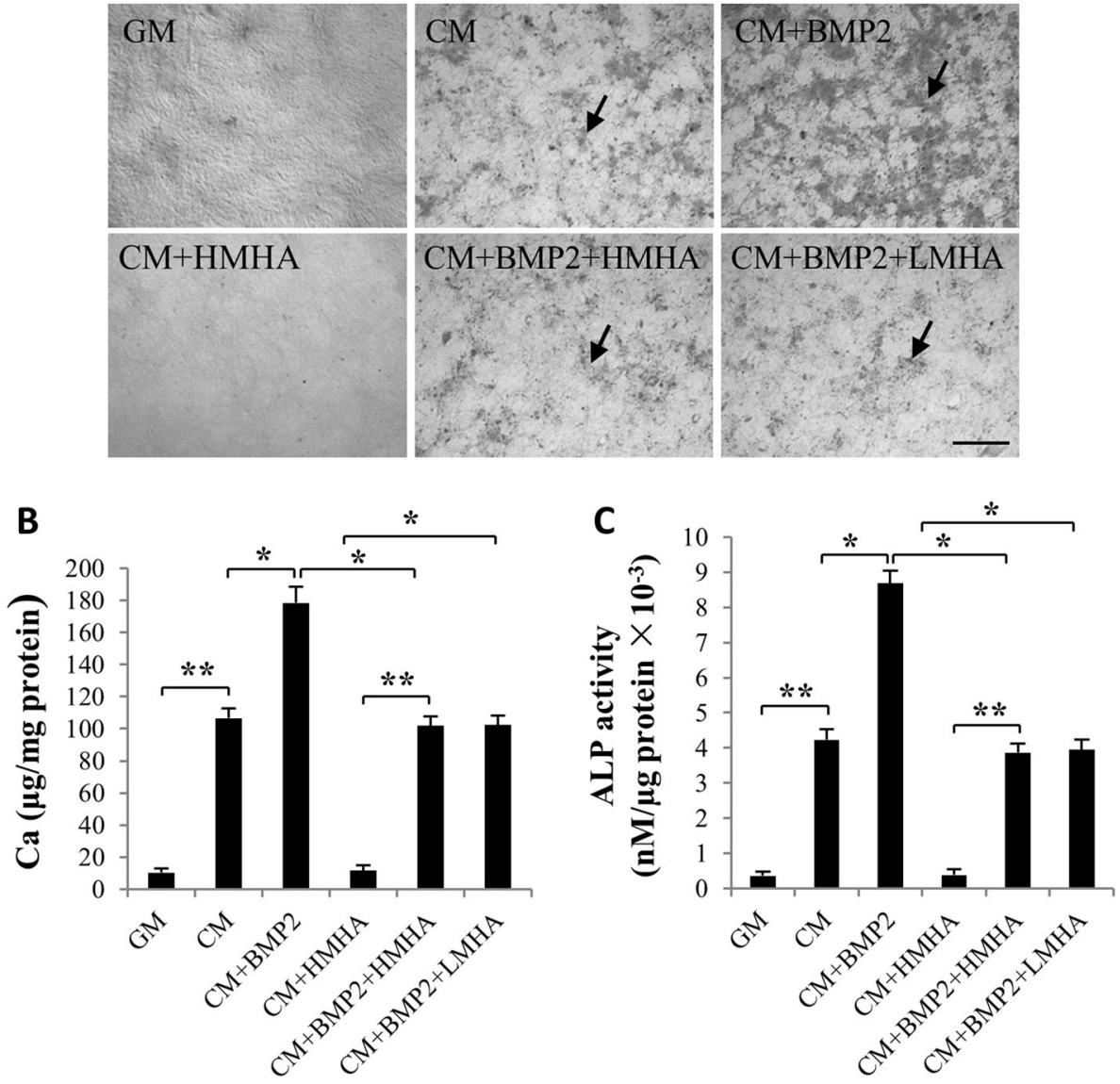

In addition, ECM not only acts as a major site for mineral deposition, but actively regulates vascular calcification. Abnormal assembly and/or remodeling of the vascular ECM may initiate ectopic calcification [41]. Previous studies have revealed that Elastin, a major elastic lamina protein produced by VSMCs, acts as a mineral nucleator to initiate vascular calcification [42, 43]. Given that HA is a main structural component of the ECM, we can not rule out the possibility that HA may directly affect the minerals' predisposition to precipitate into ECM.

In conclusion, this study identifies $\mathrm{HA}$ as a novel regulator of vascular calcification and HA negatively regulates vascular calcification involving BMP2 signaling, suggesting that HA may act as a novel potential therapeutic target for the prevention and treatment of vascular calcification. Further studies are necessary to determine whether HA regulates vascular calcification in vivo using HAS2 conditional KO mouse model.

Acknowledgements This work was supported by the National Natural Science Foundation of China (81470488, 81770280 and 81728004), the Guangdong Natural Science Foundation, China (2015A030313260, 2015A030312009 and 2016A030313226), the Science and Technology Planning Project of Guangdong Province
(2017A020215184), Undergraduate Innovation Experiment Program (201612121087) and the Scientific Research Foundation for the Returned Overseas Chinese Scholars, State Education Ministry (to JYY).

\section{Compliance with ethical standards}

Conflict of interest The authors declare that they have no conflict of interest.

\section{References}

1. Shroff R, Long DA, Shanahan C. Mechanistic insights into vascular calcification in CKD. J Am Soc Nephrol. 2013;24:179-89.

2. Vervloet M, Cozzolino M. Vascular calcification in chronic kidney disease: different bricks in the wall? Kidney Int. 2017;91:808-17.

3. Lanzer P, Boehm M, Sorribas V, Thiriet M, Janzen J, Zeller T, et al. Medial vascular calcification revisited: review and perspectives. Eur Heart J. 2014;35:1515-25.

4. Deng L, Huang L, Sun Y, Heath JM, Wu H, Chen Y. Inhibition of FOXO1/3 promotes vascular calcification. Arterioscler Thromb Vasc Biol. 2015;35:175-83.

5. Paloian NJ, Leaf EM, Giachelli CM. Osteopontin protects against high phosphate-induced nephrocalcinosis and vascular calcification. Kidney Int. 2016;89:1027-36. 
6. Kestenbaum B, Sampson JN, Rudser KD, Patterson DJ, Seliger SL, Young B, et al. Serum phosphate levels and mortality risk among people with chronic kidney disease. J Am Soc Nephrol. 2005; 16:520-8.

7. Masumoto A, Sonou T, Ohya M, Yashiro M, Nakashima Y, Okuda K, et al. Calcium overload accelerates phosphate-induced vascular calcification via Pit-1, but not the calcium-sensing receptor. J Atheroscler Thromb. 2017;24:716-24.

8. Luo G, Ducy P, McKee MD, Pinero GJ, Loyer E, Behringer RR, et al. Spontaneous calcification of arteries and cartilage in mice lacking matrix GLA protein. Nature. 1997;386:78-81.

9. Bucay N, Sarosi I, Dunstan CR, Morony S, Tarpley J, Capparelli C, et al. Osteoprotegerin-deficient mice develop early onset osteoporosis and arterial calcification. Genes Dev. 1998;12: 1260-8.

10. Fischer JW, Steitz SA, Johnson PY, Burke A, Kolodgie F, Virmani R, et al. Decorin promotes aortic smooth muscle cell calcification and colocalizes to calcified regions in human atherosclerotic lesions. Arterioscler Thromb Vasc Biol. 2004;24:2391-6.

11. Yan J, Stringer SE, Hamilton A, Charlton-Menys V, Gotting C, Muller B, et al. Decorin GAG synthesis and TGF-beta signaling mediate Ox-LDL-induced mineralization of human vascular smooth muscle cells. Arterioscler Thromb Vasc Biol. 2011;31:608-15.

12. Evanko SP, Johnson PY, Braun KR, Underhill CB, Dudhia J, Wight TN. Platelet-derived growth factor stimulates the formation of versican-hyaluronan aggregates and pericellular matrix expansion in arterial smooth muscle cells. Arch Biochem Biophys. 2001;394:29-38.

13. Vendrov AE, Madamanchi NR, Niu XL, Molnar KC, Runge M, Szyndralewiez C, et al. NADPH oxidases regulate CD44 and hyaluronic acid expression in thrombin-treated vascular smooth muscle cells and in atherosclerosis. $J$ Biol Chem. 2010;285:26545-57.

14. Matsumoto K, Li Y, Jakuba C, Sugiyama Y, Sayo T, Okuno M, et al. Conditional inactivation of Has2 reveals a crucial role for hyaluronan in skeletal growth, patterning, chondrocyte maturation and joint formation in the developing limb. Development. 2009;136:2825-35.

15. Kothapalli D, Flowers J, Xu T, Pure E, Assoian RK. Differential activation of ERK and Rac mediates the proliferative and antiproliferative effects of hyaluronan and CD44. J Biol Chem. 2008;283:31823-9.

16. Goueffic Y, Guilluy C, Guerin P, Patra P, Pacaud P, Loirand G. Hyaluronan induces vascular smooth muscle cell migration through RHAMM-mediated PI3K-dependent Rac activation. Cardiovasc Res. 2006;72:339-48.

17. Chang EJ, Kim HJ, Ha J, Kim HJ, Ryu J, Park KH, et al. Hyaluronan inhibits osteoclast differentiation via Toll-like receptor 4. J Cell Sci. 2007;120:166-76.

18. Kaneko K, Higuchi C, Kunugiza Y, Yoshida K, Sakai T, Yoshikawa $\mathrm{H}$, et al. Hyaluronan inhibits BMP-induced osteoblast differentiation. FEBS Lett. 2015;589:447-54.

19. Simpson RM, Hong X, Wong MM, Karamariti E, Bhaloo SI, Warren D, et al. Hyaluronan is crucial for stem cell differentiation into smooth muscle lineage. Stem Cells. 2016;34:1225-38.

20. Kawano M, Ariyoshi W, Iwanaga K, Okinaga T, Habu M, Yoshioka I, et al. Mechanism involved in enhancement of osteoblast differentiation by hyaluronic acid. Biochem Biophys Res Commun. 2011;405:575-80.

21. Shu B, Zhang M, Xie R, Wang M, Jin H, Hou W, et al. BMP2, but not BMP4, is crucial for chondrocyte proliferation and maturation during endochondral bone development. J Cell Sci. $2011 ; 124: 3428-40$.
22. Bostrom K, Watson KE, Horn S, Wortham C, Herman IM, Demer LL. Bone morphogenetic protein expression in human atherosclerotic lesions. J Clin Invest. 1993;91:1800-9.

23. Li X, Yang HY, Giachelli CM. BMP-2 promotes phosphate uptake, phenotypic modulation, and calcification of human vascular smooth muscle cells. Atherosclerosis. 2008;199:271-7.

24. Nakagawa Y, Ikeda K, Akakabe Y, Koide M, Uraoka M, Yutaka $\mathrm{KT}$, et al. Paracrine osteogenic signals via bone morphogenetic protein-2 accelerate the atherosclerotic intimal calcification in vivo. Arterioscler Thromb Vasc Biol. 2010;30:1908-15.

25. Hou M, Song Y, Li Z, Luo C, Ou JS, Yu H, et al. Curcumin attenuates osteogenic differentiation and calcification of rat vascular smooth muscle cells. Mol Cell Biochem. 2016;420:151-60.

26. Lu S, Liu H, Lu L, Wan H, Lin Z, Qian K, et al. WISP1 overexpression promotes proliferation and migration of human vascular smooth muscle cells via AKT signaling pathway. Eur J Pharmacol. 2016;788:90-7.

27. Song Y, Hou M, Li Z, Luo C, Ou JS, Yu H, et al. TLR4/NFkappaB/Ceramide signaling contributes to Ox-LDL-induced calcification of human vascular smooth muscle cells. Eur J Pharmacol. 2017;794:45-51.

28. Kim JH, Choi YK, Do JY, Choi YK, Ha CM, Lee SJ, et al. Estrogen-related receptor gamma plays a key role in vascular calcification through the upregulation of BMP2 expression. Arterioscler Thromb Vasc Biol. 2015;35:2384-90.

29. Byon CH, Javed A, Dai Q, Kappes JC, Clemens TL, DarleyUsmar VM, et al. Oxidative stress induces vascular calcification through modulation of the osteogenic transcription factor Runx2 by AKT signaling. J Biol Chem. 2008;283:15319-27.

30. Tang Y, Xu Q, Peng H, Liu Z, Yang T, Yu Z, et al. The role of vascular peroxidase 1 in ox-LDL-induced vascular smooth muscle cell calcification. Atherosclerosis. 2015;243:357-63.

31. Chang JR, Guo J, Wang Y, Hou YL, Lu WW, Zhang JS, et al. Intermedin1-53 attenuates vascular calcification in rats with chronic kidney disease by upregulation of alpha-Klotho. Kidney Int. 2016;89:586-600.

32. Lin ME, Chen TM, Wallingford MC, Nguyen NB, Yamada S, Sawangmake C, et al. Runx2 deletion in smooth muscle cells inhibits vascular osteochondrogenesis and calcification but not atherosclerotic lesion formation. Cardiovasc Res. 2016;112:606-16.

33. Lovekamp J, Vyavahare N. Periodate-mediated glycosaminoglycan stabilization in bioprosthetic heart valves. J Biomed Mater Res. 2001;56:478-86.

34. Ohri R, Hahn SK, Hoffman AS, Stayton PS, Giachelli CM. Hyaluronic acid grafting mitigates calcification of glutaraldehydefixed bovine pericardium. J Biomed Mater Res A. 2004;70:328-34.

35. Miyazaki T, Miyauchi S, Tawada A, Anada T, Matsuzaka S, Suzuki O. Oversulfated chondroitin sulfate-E binds to BMP-4 and enhances osteoblast differentiation. J Cell Physiol. 2008;217:769-77.

36. Nagy N, Freudenberger T, Melchior-Becker A, Rock K, Ter Braak M, Jastrow $\mathrm{H}$, et al. Inhibition of hyaluronan synthesis accelerates murine atherosclerosis: novel insights into the role of hyaluronan synthesis. Circulation. 2010;122:2313-22.

37. Chai S, Chai Q, Danielsen CC, Hjorth P, Nyengaard JR, Ledet T, et al. Overexpression of hyaluronan in the tunica media promotes the development of atherosclerosis. Circ Res. 2005;96:583-91.

38. Matsubara T, Kida K, Yamaguchi A, Hata K, Ichida F, Meguro H, et al. BMP2 regulates Osterix through Msx2 and Runx2 during osteoblast differentiation. J Biol Chem. 2008;283:29119-25.

39. Zhu W, Boachie-Adjei O, Rawlins BA, Frenkel B, Boskey AL, Ivashkiv $\mathrm{LB}$, et al. A novel regulatory role for stromal-derived 
factor-1 signaling in bone morphogenic protein-2 osteogenic differentiation of mesenchymal $\mathrm{C} 2 \mathrm{C} 12$ cells. J Biol Chem. 2007;282:18676-85.

40. Derwall M, Malhotra R, Lai CS, Beppu Y, Aikawa E, Seehra JS, et al. Inhibition of bone morphogenetic protein signaling reduces vascular calcification and atherosclerosis. Arterioscler Thromb Vasc Biol. 2012;32:613-22.

41. Khavandgar Z, Roman H, Li J, Lee S, Vali H, Brinckmann J, et al. Elastin haploinsufficiency impedes the progression of arterial calcification in MGP-deficient mice. $\mathrm{J}$ Bone Miner Res. 2014;29:327-37.

42. Seligman M, Eilberg RF, Fishman L. Mineralization of elastin extracted from human aortic tissues. Calcif Tissue Res. 1975;17:229-34.

43. Basalyga DM, Simionescu DT, Xiong W, Baxter BT, Starcher BC, Vyavahare NR. Elastin degradation and calcification in an abdominal aorta injury model: role of matrix metalloproteinases. Circulation. 2004;110:3480-7. 